oncology/bone marrow transplant and haematology ward (Starlight) in 2016 showed that only 33\% of fluid prescriptions for PN were written before 6pm. During 2016-2017 Starlight ward piloted a new prescribing system whereby nurses administered PN directly from the prescription used to order PN from the aseptic unit. An audit in early 2018 showed that PN was routinely set-up, checked and started by 1800 hours, nurses were able to plan their time effectively and oncall doctors were only involved if patient condition warranted review. In March 2018 the pilot was replaced with similar redesigned process.

Aim To eliminate the process of prescribing volumes and flow rates for PN on fluid prescriptions. To trial a new PN prescription process on one ward, refine and improve as necessary then adopt across the whole of the hospital.

Methods On Starlight ward in March 2018 a new process for prescribing and administering PN was implemented. Nurses used the prescription for ordering $\mathrm{PN}$ from the aseptic unit plus the product insert to set-up, start and sign for administration. A new aseptic unit prescription was created, nursing training was provided and written guidance was issued for nurses on how to use perform set-up checks. PN prescriptions were kept on the ward. Stickers that highlighted the patient required $\mathrm{PN}$ were placed onto fluid prescriptions to prevent PN inadvertently not being administered.

Results All patients prescribed PN on Starlight ward received it as expected. As nurses had flexibility in PN set-up time once the product was on the ward, patient routine and preference (e.g. going out for day leave) was increasingly taken into account leading to PN often starting after 18 hours. One minor incident relating to stickers occurred which did not affect the patient. Nursing feedback was very positive. By eliminating transcribing, the process was perceived as safer. In July the trial was evaluated and one change was made to the prescription to allow clearer adjustment of PN rate/volume after the infusion began. The prescribing process was implemented on a surgical ward in August and will be rolled out across the rest of the hospital pending the outcome.

Conclusion Simplifying the prescribing process meant PN was administered at a time that suited the patient and nurse. Nurse satisfaction was improved and avoiding transcription was perceived as safer. The process will be rolled out in stages to the rest of the hospital.

\section{P024 THE USE OF PARENTERAL NUTRITION IN PAEDIATRIC HAEMATOLOGY AND ONCOLOGY PATIENTS}

${ }^{1}$ Sam Whiting, ${ }^{2}$ Heather Weerdenburg. ${ }^{1}$ Bristol Royal Hospital for Children; ${ }^{2}$ Bristol Children's Hospital

\subsection{6/archdischild-2019-nppc.34}

Introduction A trust wide Parenteral Nutrition (PN) guideline is available to advise the initiating, monitoring and stopping of PN. ${ }^{1}$ Following an increase in demand on the paediatric oncology, haematology and bone marrow transplant (BMT) ward the pharmacy and dietician team decided to audit how we use PN against these guidelines.

Objectives Is PN being started and stopped appropriately according to the nutrition guidelines? Are patients being monitored on PN appropriately? Are there alternative sources of feeding that could be initiated by enteral route prior to starting PN?
Methodology The pharmacy dispensing system was used to trace which BMT, haematology or oncology patients required parenteral nutrition. A combination of the medical notes and the electronic Medway system for those patients' notes was used to collect data. Data was collected over a 12 month period from March 2017 until February 2018, a total of 29 patients were identified and audited.

Results Alternative feeding routes to $\mathrm{PN}$ were deemed inappropriate in all 29 patients. A full plan had only been recorded in the patient notes in just 4\% (1/29) of cases. Biochemistry was routinely provided prior to initiating $\mathrm{PN}$ but there was a failure to monitor patients needing long term biochemistry with only $11 \%(1 / 9)$ of patients having long term bloods reported. Only 38\% (10/26) of patients had PN discontinued when the patient reached two-thirds of their target enteral intake.

Conclusion A plan for PN is often omitted in the medical notes. There should be an expected duration, a desired outcome, IV access and a plan around what other (if any) nutrition can be given alongside. We plan to develop a PN plan proforma which can be used to stick into the notes which prompts the medical team responsible to enter this information. There is a lack of timely long term biochemistry bloods on those patients that have PN for longer than a month. This is important clinically because long term $\mathrm{PN}$ patients can develop deficiency in micronutrients which need replacement. We hope that educating the medical and nursing teams about this aspect of the clinical guideline will improve our practice. Lastly, the aim of PN must be to establish nutritional requirements where otherwise calorie input would not be met. Stopping early will lead to a calorie deficit and stopping too late would mean unnecessary extra clinical risk and potential inpatient stay. There were several instances where patients would have been discharged because they were otherwise clinically well but feeds were not adequate to stop PN. Other times PN is continued at $25 \%$ of requirements, where we should be stopping as soon as patients are established on $66 \%$ of oral calorie intake. This should be part of the wider team education about PN

\section{REFERENCE}

1. Phipps A. April 2016, Total Parenteral Nutrition Guideline. Bristol Children's Hospital.

\section{P025 CLINICAL PEARL; TREATING INFANT BOTULISM ON A PAEDIATRIC INTENSIVE CARE UNIT (PICU)}

Caroline Dalton. Imperial College Healthcare NHS Trust

10.1136/archdischild-2019-nppc.35

Problem A call was received out of hours by the specialist PICU pharmacist (SP). A five month old baby with rapidly spreading paralysis of unknown cause had been admitted to the unit. A toxin had been extracted from the stool culture and tested on mice. Within hours all mice had died, confirming a positive result for Botulism toxin. The SP was asked to obtain an urgent supply of Human Botulism Anti-Toxin however the only worldwide manufacturer/supplier, the Infant Botulism Treatment and Prevention Program (IBTPP), is based in California. ${ }^{1}$ BabyBIG, Botulism Immune Globulin Intravenous (Human) (BIG-IV), is an orphan drug that consists of human-derived anti-botulism toxin antibodies that is approved by the U.S. Food and Drug Administration for the 